\title{
Divided attention reduces resistance to distraction at encoding but not retrieval
}

\author{
Jennifer C. Weeks ${ }^{1,2}$ • Lynn Hasher ${ }^{1,2}$
}

Published online: 29 November 2016

(C) Psychonomic Society, Inc. 2016

\begin{abstract}
Older adults show implicit memory for previously seen distraction, an effect attributed to poor attentional control. It is unclear whether this effect results from lack of control over encoding during the distraction task, lack of retrieval constraint during the test task, or both. In the present study, we simulated poor distraction control in young adults using divided attention at encoding, at retrieval, at both times, or not at all. The encoding task was a 1-back task on pictures with distracting superimposed letter strings, some of which were words. The retrieval task was a word fragment completion task testing implicit memory for the distracting words. Attention was divided using an auditory odd digit detection task. Dividing attention at encoding, but not at retrieval, resulted in significant priming for distraction, which suggests that control over encoding processes is a primary determinant of distraction transfer in populations with low inhibitory control (e.g. older adults).
\end{abstract}

Keywords Priming $\cdot$ Distraction $\cdot$ Divided attention · Inhibition

Across domains of cognitive psychology, from attention and memory to decision making, an individual's ability to exercise cognitive control and ignore irrelevant information is

Jennifer C. Weeks

jennifer.weeks@utoronto.ca

Lynn Hasher

hasher@psych.utoronto.ca

1 Department of Psychology, University of Toronto, 100 St. George Street, Toronto, Ontario M5S 3G3, Canada

2 Rotman Research Institute, Baycrest, Toronto, Ontario, Canada M6A 2E1 consistently observed as a limiting factor to performance. Suppression of irrelevant information allows people to focus on the task at hand, respond quickly to targets, avoid making errors, and selectively recall items within their correct contexts. When the ability to ignore irrelevant information is diminished, as it is in aging (e.g. Hasher, Zacks, \& May, 1999) and when young adults are doing a challenging divided attention (DA) task (de Fockert, Rees, Frith, \& Lavie, 2001), there are serious implications for performance on a broad range of cognitive tasks (e.g. Weeks \& Hasher, 2014).

Although a lack of cognitive control is detrimental to performance on many tasks, recent work demonstrates that there are some benefits to reduced regulation, at least when distracters in one task become relevant in a subsequent task. In one study, young and older participants performed a selective attention task on pictures, ignoring superimposed distracter words, and older adults outperformed young adults on a subsequent word fragment completion task in which previous distracter words served as solutions to some fragments (Rowe et al., 2006). Older adults' implicit knowledge of distraction has now been shown to transfer to a variety of other test tasks including cued recall (Campbell, Hasher, \& Thomas, 2010; Weeks, Biss, Murphy, \& Hasher, 2016), prospective memory (Lourenço \& Maylor, 2015), and free recall (Biss, Ngo, Hasher, Campbell, \& Rowe, 2013), all without participants reporting any awareness of the relevance of the distracters. It is currently unclear whether the observed tacit transfer of distraction to later tasks is related to a lack of attentional control at encoding, retrieval, or both. We addressed this question in the present study using divided attention (DA) to simulate reduced cognitive control in young adults.

Reducing young adults' attention by assigning a secondary task during encoding has been shown to decrease both explicit recall (Craik, Govoni, Naveh-Benjamin, \& Anderson, 1996) and implicit conceptual priming for targets (Mulligan, 1997, 
1998; Mulligan \& Hartman, 1996), as well as to increase behavioural interference from distracter stimuli (de Fockert et al., 2001) and false memories on the Deese/RoedigerMcDermott task (Peters et al., 2008). The effects of divided attention at encoding in young adults are strikingly similar to the pattern of cognitive impairment commonly observed among older adults, who show increased processing of distracters relative to their young counterparts (Campbell, Grady, Ng, \& Hasher, 2012; de Fockert, Ramchurn, Van Velzen, Bergström, \& Bunce, 2009; Haring et al., 2013; May, 1999), accompanied by impaired recall of target items (e.g. Gazzaley, Cooney, Rissman, \& D'Esposito, 2005).

At retrieval, cognitive or inhibitory control is required to select the correct items from memory and overcome interference from similar, competing stimuli (e.g. Healey, Ngo, \& Hasher, 2014). DA at retrieval has been shown to consume attentional resources, as evidenced by large costs to the secondary task (e.g. Craik et al., 1996) and costs to retrieval in circumstances in which attentional resources are in high demand, such as when retrieval demands recollective processes (Hicks \& Marsh, 2000; Lozito \& Mulligan, 2006) or competes for the same cognitive processes as the secondary task (Fernandes \& Moscovitch, 2000, 2003; Fernandes, Wammes, Priselac, \& Moscovitch, 2016). Although DA at retrieval typically does not impair explicit or implicit memory to the same degree as DA at encoding (Lozito \& Mulligan, 2010; Naveh-Benjamin, Craik, Perretta, \& Tonev, 2000; Troyer \& Craik, 2000), there is at least indirect evidence that inhibitory control processes are reduced by the addition of a secondary task at test. During an old/new recognition task, both young adults under DA conditions and older adults under full attention (FA) conditions show a bias toward responding "old" when test items are presented simultaneously with familiar, to-be-ignored distracters (Anderson, Jacoby, Thomas, $\&$ Balota, 2011). This result, in combination with similar findings elsewhere in the literature (e.g. Ste-Marie \& Jacoby, 1993; Jacoby, 1991; Jacoby, Woloshyn, \& Kelley, 1989), suggests that DA reduces top-down constraint over retrieval, allowing irrelevant stimuli to be spontaneously retrieved.

In the present study, young adult participants saw verbal distraction in the context of a target task and implicit memory for that distraction was tested after a delay. We simulated an attentional control deficit by assigning participants a concurrent auditory monitoring task during encoding, retrieval, or both. Control participants did not do the concurrent task at either encoding or retrieval. Based on evidence that dividing attention disrupts controlled processes while leaving automatic processes relatively intact (Craik et al., 1996; Jacoby, 1991; Schmitter-Edgecombe, 1999), we reasoned that dividing attention in a target task that included distraction would reduce active inhibitory processes involved in selective attention and retrieval constraint, thereby increasing automatic encoding of distracters and automatic retrieval of the recent past. We were interested in which simulated deficit condition, if any, would show transfer of distraction like that seen in older adults (e.g. Rowe et al., 2006).

\section{Method}

\section{Participants}

Ninety-six young adults (age 17-25) were recruited from an introductory psychology class and randomly assigned to one of the four conditions. Sample size was based on the average sample size reported in previous studies showing age differences in priming for distraction (e.g. Campbell et al., 2012; Rowe et al., 2006). The Research Ethics Board at University of Toronto approved the study procedure and participants provided written consent prior to starting the experiment. All participants reported learning English before age 4 and did not identify as having an East Asian cultural background (see Amer, Ngo, \& Hasher, 2016 for evidence of cultural effects on selective attention and subsequent memory). Demographic data for each group are presented in Table 1. One participant indicated being fully aware that some distracter words from the selective attention task served as solutions in the word fragment task, and they were replaced prior to data analysis.

\section{Materials}

Picture stimuli Sixty unique pictures from Snodgrass \& Vanderwart (1980) were used as targets in the initial selective attention task. Pictures were coloured red and slightly rotated from the vertical axis, as in Rowe et al. (2006).

Word stimuli Two lists of 15 critical words were matched on word length $(M=6$ letters $)$ and number of letters in their respective word fragments ( $M=3.4$ letters). Half the participants in each condition were exposed to distracter words from one list in the selective attention task and the other half were exposed to distracter words from the other list. Distracter words and target pictures were pseudo-randomly paired in the selective attention task so that none of the picture-word

Table 1 Demographic information by condition

\begin{tabular}{llll}
\hline Condition & Age & Education & Vocabulary \\
\hline Full attention & $19.5(2.3)$ & $13.4(1.6)$ & $31.0(3.5)$ \\
DA at Encoding & $18.5(1.1)$ & $12.9(1.5)$ & $30.1(4.2)$ \\
DA at Retrieval & $18.5(0.9)$ & $13.0(1.3)$ & $28.9(3.8)$ \\
DA at Both & $18.4(1.1)$ & $12.5(0.9)$ & $30.2(3.5)$ \\
\hline
\end{tabular}

Standard deviations are in parentheses 
pairs were semantically related. In the subsequent word fragment task, participants were shown all word fragments from both lists (one list primed, the other unprimed), along with 15 easy word fragments included to conceal the connection between the selective attention and word fragment tasks.

Auditory stimuli Digits were presented through headphones at a rate of one digit every $2 \mathrm{~s}$ at a volume that was comfortable for the participant.

\section{Procedure}

The study procedure consisted of three phases: an initial selective attention task with distraction, a 7-min delay, and an implicit fragment completion task. The auditory digit task was performed several times throughout the experimental procedure, at various times depending on the assigned condition. Prior to the selective attention task, participants in the DA at Encoding conditions completed a 2-min practice run of the auditory digit task to familiarize themselves with the task.

Selective attention task Participants saw a series of pictures shown for $1000 \mathrm{~ms}$ each with a 500-ms inter-stimulus interval, and were asked to press the "YES" key whenever they saw a picture repeat and to press "NO" otherwise. The task began with five pictures containing no distraction, and then eight pictures superimposed with random letter strings. Then, a series of 53 pictures was presented superimposed with either critical words that would later serve as solutions to word fragments (15), filler words (15), or random letter strings (23). The task ended with a recency buffer of eight pictures superimposed with random letter strings. Target trials on which participants had to respond "YES" occurred every six trials on average and never contained a critical word in order to avoid the increased perceptual priming that is seen for distracters appearing alongside infrequent targets (Spataro, Mulligan, \& Rossi-Arnaud, 2013). Participants assigned to DA at Encoding conditions performed the auditory digit detection task concurrently with the selective attention task, saying "Now" aloud each time they heard three odd digits in a row. Auditory target trials occurred every eight trials on average.

Filled delay period All participants, including those in the Full Attention (FA) condition, performed the auditory digit detection task for 2 min during the filled delay interval following the selective attention task. For the remainder of the 7-min delay period, they performed an equation fragment completion task.

Word fragment completion task After the delay, participants in all conditions were asked to complete a series of word fragments with the first word that came to mind. They had
$4 \mathrm{~s}$ to produce an oral response to each word fragment. After five initial buffer fragments, participants saw 15 fragments from the primed list, 15 fragments from the unprimed list, and 15 easy filler fragments in alternating order. Participants assigned to the DA at Retrieval conditions performed the auditory digit detection task concurrently with the word fragment completion task, pressing the Enter key when they heard three odd digits in a row so as not to interfere with the spoken word responses. Finally, participants completed a graded awareness questionnaire probing their awareness of the connection between the tasks, and were then debriefed and assigned a partial course credit.

\section{Results}

\section{Selective attention task}

Accuracy on the 1-back task was calculated as percentage of hits minus false alarms (Table 2). Accuracy was entered into a 2 (attention at encoding: FA or DA) X 2 (attention at retrieval: FA or DA) ANOVA. The sole reliable effect was a main effect of attention at encoding such that participants in the DA at Encoding conditions performed worse on the selective attention task than those whose attention was not divided during distracter presentation, $F(1,92)=13.76, p<.001, \eta_{\mathrm{p}}{ }^{2}=.13$. Performance on the digit detection task did not differ between the two groups that performed it during the 1-back task (Table 2; $t(46)<1$ ).

\section{Priming for distraction}

Priming for distracter words was calculated as an individual's rate of primed fragment completion minus their group's average unprimed fragment completion, as is commonly done in the implicit memory literature (e.g. Rowe et al., 2006). Completion rates for unprimed and filler fragments did not differ across conditions, $F \mathrm{~s}<1$. Raw scores on the word fragment task are reported in Table 3. Priming scores were submitted to a $2 \times 2$ ANOVA, which confirmed only a main effect of attention at encoding such that participants whose attention was divided at encoding showed greater priming for distraction than those whose attention was not divided, $F(1,92)=6.73, p=.011, \eta_{\mathrm{p}}{ }^{2}=.07$. Dividing attention during the word fragment test evidently had no effect on priming for distraction. Both DA at Encoding groups had average priming scores that, although small, differed reliably from zero (DA at Encoding: $M=3.9 \%, \mathrm{SD}=9.3 \%, t(23)=2.56, p=.05$; DA at Both: $M=3.3 \%, \mathrm{SD}=5.2 \%, t(23)=3.14, p=.005)$. Priming scores for the full attention at encoding conditions did not differ from zero (FA: $M=-0.8 \%, \mathrm{SD}=5.4 \%, t(23)<1$; DA at Retrieval: $M=0.0 \%, \mathrm{SD}=9.4 \%, t(23)<1)$. The two groups that performed the divided attention task concurrently with the 
Table 2 Performance on selective attention task (\%hits minus \%false alarms)

\begin{tabular}{llr}
\hline Condition & Accuracy on 1-back task & $\begin{array}{c}\text { Accuracy on secondary } \\
\text { digit detection task }\end{array}$ \\
\hline Full attention & $81.4 \%(25.6 \%)$ & N/A (FA at Encoding) \\
DA at Encoding & $61.8 \%(20.2 \%)$ & $75.7 \%(21.1 \%)$ \\
DA at Retrieval & $82.2 \%(20.7 \%)$ & N/A (FA at Encoding) \\
DA at Both & $68.7 \%(20.4 \%)$ & $71.0 \%(30.2 \%)$ \\
\hline
\end{tabular}

Standard deviations are in parentheses

fragment completion task did not differ on accuracy on the auditory digit detection task (Table $3 ; t(46)<1$ ), suggesting that the digit task did not become easier for the participants in the DA at the Both condition who had slightly more practice.

\section{Discussion}

Older but not younger adults have been shown to transfer knowledge of previous distraction to new tasks. The presumed mechanism for these transfer effects is reduced attentional control, potentially resulting from an inhibitory deficit that is characteristic of older adults (Hasher \& Zacks, 1988), but the stage at which these effects are exerted is currently unknown. Here, we simulated impaired attentional control in healthy young adults by dividing attention during a distraction task and/or during a transfer task and observing the consequences for implicit priming for distracters. We hypothesized that, if reduced control over distracters at encoding contributes to the acquisition of distracting information and its transfer to new tasks, then there would be a main effect of dividing attention at encoding, and this result was found to be reliable. We also hypothesized that, if failure to inhibit spontaneous retrieval of previously seen distracters at the time of test contributes to the transfer effect, then we would observe a main effect of dividing attention at retrieval, but we did not find evidence of this effect. Finally, if reduced inhibitory control at both stages has an additive effect, we expected to see an interaction between dividing attention at encoding and dividing attention at retrieval such that the greatest priming for distraction would be found in the DA at the Both condition, but this additive effect was not observed. Thus, the origin of the transfer of distraction phenomenon appears to be increased encoding of irrelevant information under conditions of low attentional control.

DA at encoding has been shown to disrupt effortful and elaborative encoding processes during intentional study, which has a profound detrimental effect on subsequent explicit memory for target items (e.g. Craik et al., 1996). However, the present findings demonstrate that DA at encoding has the additional consequence of reducing attentional control and increasing encoding of non-target items, which is likely to create interference between target items and encoded distracters (e.g. Postman \& Underwood, 1973). This interference may be another contributing factor to the widely reported disruptive effects of DA at encoding.

Under the logic that DA at the time of retrieval consumes attentional resources that cannot then be used to constrain retrieval to relevant items, we anticipated that young adults under DA during the transfer task would automatically retrieve previously seen distracter words and solve more word fragments using previously distracting words. However, we observed no effect of dividing attention at retrieval, even in the DA condition in which participants decidedly had encoded the distracting words (i.e. the DA at the Both condition). The failure to find an effect of DA at retrieval on perceptual priming echoes previous findings that implicit retrieval is an automatic process that is unaffected by limitations on attentional resources (e.g. Lozito and Mulligan, 2010). Given that the DA at Encoding group and the DA at the Both group showed

Table 3 Performance on word fragment task

\begin{tabular}{|c|c|c|c|c|}
\hline \multirow[t]{2}{*}{ Condition } & \multicolumn{3}{|c|}{ Percentage solved word fragments } & \multirow{2}{*}{$\begin{array}{l}\text { Accuracy on secondary } \\
\text { digit detection task }\end{array}$} \\
\hline & Primed & Unprimed & Filler & \\
\hline Full attention & $8.9 \%(5.4 \%)$ & $9.7 \%(8.6 \%)$ & $51.9 \%(17.9 \%)$ & N/A (FA at retrieval) \\
\hline DA at Encoding & $11.7 \%(9.3 \%)$ & $7.8 \%(5.1 \%)$ & $48.1 \%(14.7 \%)$ & N/A (FA at retrieval) \\
\hline DA at Retrieval & $10.3 \%(9.2 \%)$ & $9.2 \%(7.8 \%)$ & $50.8 \%(17.1 \%)$ & $48.2 \%(20.6 \%)$ \\
\hline DA at Both & $10.3 \%(5.2 \%)$ & $6.9 \%(8.2 \%)$ & $46.1 \%(19.1 \%)$ & $52.3 \%(17.5 \%)$ \\
\hline
\end{tabular}

Standard deviations are in parentheses 
equivalent amounts of priming for distraction, it is likely that priming on the word fragment task is a direct reflection of the extent to which distracters were processed during encoding (Schacter, 1990; Tulving \& Schacter, 1990). An interesting follow-up to this study could examine the effect of DA at retrieval during an explicit task measuring transfer of distraction (e.g. Biss et al., 2013), as explicit memory may be more susceptible to effects of unconstrained retrieval.

One of the goals of this research is to characterize the mechanism of the distraction transfer effect that has been shown to benefit memory in older adults (e.g. Amer \& Hasher, 2014; Rowe et al., 2006; Weeks et al., 2016). The presence of significant priming for distraction in the DA at Encoding conditions confirms that lack of efficient control over distraction is an important component of this effect. However, the magnitude of distracter priming in the groups of young adults with simulated encoding deficits tested here is approximately one-third the level of distracter priming previously observed among older adults (e.g. Biss, Weeks, \& Hasher, 2012; Campbell et al., 2012). This comparison, although quite indirect, suggests that none of the DA manipulations employed in the current study produce a perfect simulation of aging, either because older adults' attentional control during encoding is even more dysregulated than that of DA young adults or because older adults are more affected by attentional impairments at retrieval than are DA young adults. At Encoding, there are a number of factors other than DA that have been shown to increase processing of distracters, including positive mood (e.g. Biss \& Hasher, 2011) and being tested at off-peak times of day (Rowe et al., 2006), both of which may be more common among older adults, who are often happier and more morning-type than young adults (Biss \& Hasher, 2012); these factors may be additive with or aggravate the effects of age-related attentional dysregulation, producing the large distracter priming effects previously seen in older adult samples. Another, non-mutually exclusive possibility is that older adults' retrieval control processes are weaker than even those of young adults under DA at retrieval. Indeed, older adults show greater susceptibility to interference (Ikier, Yang, \& Hasher, 2008) and a reduced ability to resolve interference at retrieval compared to young adults (Healey, Hasher, \& Campbell, 2013). Also, older adults show retrieval impairments that are not typically observed in young adults under DA, such as higher rates of false recognition for associates of target words (Budson, Sullivan, Daffner, \& Schacter, 2003), and a greater influence of misleading stimuli at retrieval (Jacoby, Bishara, Hessels, \& Toth, 2005), suggesting that retrieval in older adults may be affected by factors other than a simple reduction of attentional resources. Further work is needed to characterize the complex changes in both attention control and retrieval processes that occur in old age.

The literature on distraction transfer shows that unattended stimuli from the past can influence memory, and the present study extends this line of work by showing that distracters encoded under conditions of low attentional control (here, divided attention) can influence subsequent memory performance in young adults. We failed to show evidence that memory is more likely to be influenced by previous distracters under conditions of low retrieval control, but further empirical work is required to fully characterize how deficient retrieval processes may contribute to the memory benefit conferred by previous distraction.

Author Note The authors would like to thank lab manager Elizabeth Howard for her assistance in data collection. This work was funded by National Science and Engineering Research Council of Canada (NSERC) grant 487235 to L. Hasher and NSERC Alexander Graham Bell Canada Graduate Scholarship (Doctoral) to J.C. Weeks

\section{References}

Amer, T., \& Hasher, L. (2014). Conceptual processing of distractors by older but not younger adults. Psychological Science, 25(12), 22522258.

Amer, T., Ngo, K. W., \& Hasher, L. (2016). Cultural differences in visual attention: Implications for distraction processing. British Journal of Psychology.

Anderson, B. A., Jacoby, L. L., Thomas, R. C., \& Balota, D. A. (2011). The effects of age and divided attention on spontaneous recognition. Memory \& Cognition, 39(4), 725-735.

Biss, R. K., \& Hasher, L. (2011). Delighted and distracted: Positive affect increases priming for irrelevant information. Emotion, 11(6), 14741478.

Biss, R. K., \& Hasher, L. (2012). Happy as a lark: Morning-type younger and older adults are higher in positive affect. Emotion, 12(3), 437441.

Biss, R. K., Ngo, K. J., Hasher, L., Campbell, K. L., \& Rowe, G. (2013). Distraction can reduce age-related forgetting. Psychological Science, 24(4), 448-455.

Biss, R. K., Weeks, J. C., \& Hasher, L. (2012). Happily distracted: Mood and a benefit of attention dysregulation in older adults. Frontiers in Psychology, 3.

Budson, A. E., Sullivan, A. L., Daffner, K. R., \& Schacter, D. L. (2003). Semantic versus phonological false recognition in aging and Alzheimer's disease. Brain and Cognition, 51(3), 251-261.

Campbell, K. L., Grady, C. L., Ng, C., \& Hasher, L. (2012). Age differences in the frontoparietal cognitive control network: Implications for distractibility. Neuropsychologia, 50(9), 2212-2223.

Campbell, K. L., Hasher, L., \& Thomas, R. C. (2010). Hyper-binding: A unique age effect. Psychological Science, 21(3), 399-405.

Craik, F. I., Govoni, R., Naveh-Benjamin, M., \& Anderson, N. D. (1996). The effects of divided attention on encoding and retrieval processes in human memory. Journal of Experimental Psychology: General, 125(2), 159-180.

de Fockert, J. W., Ramchurn, A., Van Velzen, J., Bergström, Z., \& Bunce, D. (2009). Behavioral and ERP evidence of greater distractor processing in old age. Brain Research, 1282, 67-73.

de Fockert, J. W., Rees, G., Frith, C. D., \& Lavie, N. (2001). The role of working memory in visual selective attention. Science, 291(5509), 1803-1806.

Fernandes, M. A., \& Moscovitch, M. (2000). Divided attention and memory: Evidence of substantial interference effects at retrieval and encoding. Journal of Experimental Psychology: General, 129(2), $155-176$. 
Fernandes, M. A., \& Moscovitch, M. (2003). Interference effects from divided attention during retrieval in younger and older adults. Psychology and Aging, 18(2), 219-230.

Fernandes, M. A., Wammes, J. D., Priselac, S., \& Moscovitch, M. (2016). Interfering with free recall of words: Detrimental effects of phonological competition. Neuropsychologia.

Gazzaley, A., Cooney, J. W., Rissman, J., \& D’Esposito, M. (2005). Topdown suppression deficit underlies working memory impairment in normal aging. Nature Neuroscience, 8(10), 1298-1300.

Haring, A. E., Zhuravleva, T. Y., Alperin, B. R., Rentz, D. M., Holcomb, P. J., \& Daffner, K. R. (2013). Age-related differences in enhancement and suppression of neural activity underlying selective attention in matched young and old adults. Brain Research, 1499, 69-79.

Hasher, L., \& Zacks, R. T. (1988). Working memory, comprehension, and aging: A review and a new view. Psychology of Learning and Motivation, 22, 193-225.

Hasher, L., Zacks, R. T., \& May, C. P. (1999). Inhibitory control, circadian arousal, and age. In D. Gopher \& A. Koriat (Eds.), Attention \& performance, XVII, cognitive regulation of performance: Interaction of theory and application (pp. 653-675). Cambridge, MA: MIT Press.

Healey, M. K., Hasher, L., \& Campbell, K. L. (2013). The role of suppression in resolving interference: Evidence for an age-related deficit. Psychology and Aging, 28(3), 721-728.

Healey, K. M., Ngo, K. W. J., \& Hasher, L. (2014). Below-baseline suppression of competitors during interference resolution by younger but not older adults. Psychological Science, 25(1), 145-151.

Hicks, J. L., \& Marsh, R. L. (2000). Toward specifying the attentional demands of recognition memory. Journal of Experimental Psychology: Learning, Memory, and Cognition, 26(6), 1483-1498.

Ikier, S., Yang, L., \& Hasher, L. (2008). Implicit proactive interference, age, and automatic versus controlled retrieval strategies. Psychological Science, 19(5), 456-461.

Jacoby, L. L. (1991). A process dissociation framework: Separating automatic from intentional uses of memory. Journal of Memory and Language, 30(5), 513-541.

Jacoby, L. L., Bishara, A. J., Hessels, S., \& Toth, J. P. (2005). Aging, subjective experience, and cognitive control: Dramatic false remembering by older adults. Journal of Experimental Psychology: General, 134(2), 131-148.

Jacoby, L. L., Woloshyn, V., \& Kelley, C. (1989). Becoming famous without being recognized: Unconscious influences of memory produced by dividing attention. Journal of Experimental Psychology: General, 118(2), 115-125.

Lourenço, J. S., \& Maylor, E. A. (2015). When distraction holds relevance: A prospective memory benefit for older adults. International Journal of Environmental Research and Public Health, 12(6), 6523-6541.

Lozito, J. P., \& Mulligan, N. W. (2006). Exploring the role of attention during memory retrieval: Effects of semantic encoding and divided attention. Memory \& Cognition, 34(5), 986-998.

Lozito, J. P., \& Mulligan, N. W. (2010). Exploring the role of attention during implicit memory retrieval. Journal of Memory and Language, 63(3), 387-399.
May, C. P. (1999). Synchrony effects in cognition: The costs and a benefit.Psychonomic. Bulletin and Review, 6(1), 142-147.

Mulligan, N. W. (1997). Attention and implicit memory tests: The effects of varying attentional load on conceptual priming. Memory \& Cognition, 25(1), 11-17.

Mulligan, N. W. (1998). The role of attention during encoding in implicit and explicit memory. Journal of Experimental Psychology: Learning, Memory, and Cognition, 24(1), 27-47.

Mulligan, N. W., \& Hartman, M. (1996). Divided attention and indirect memory tests. Memory \& Cognition, 24(4), 453-465.

Naveh-Benjamin, M., Craik, F. I., Perretta, J. G., \& Tonev, S. T. (2000). The effects of divided attention on encoding and retrieval processes: The resiliency of retrieval processes. The Quarterly Journal of Experimental Psychology: Section A, 53(3), 609-625.

Peters, M. J., Jelicic, M., Gorski, B., Sijstermans, K., Giesbrecht, T., \& Merckelbach, H. (2008). The corrective effects of warning on false memories in the DRM paradigm are limited to full attention conditions. Acta Psychologica, 129(2), 308-314.

Postman, L., \& Underwood, B. J. (1973). Critical issues in interference theory. Memory \& Cognition, 1(1), 19-40.

Rowe, G., Valderrama, S., Hasher, L., \& Lenartowicz, A. (2006). Attentional disregulation: A benefit for implicit memory. Psychology and Aging, 21(4), 826-830.

Schacter, D. L. (1990). Perceptual representation systems and implicit memory. Annals of the New York Academy of Sciences, 608(1), $543-571$.

Schmitter-Edgecombe, M. (1999). Effects of divided attention on perceptual and conceptual memory tests: An analysis using a processdissociation approach. Memory \& Cognition, 27(3), 512-525.

Snodgrass, J. G., \& Vanderwart, M. (1980). A standardized set of 260 pictures: Norms for name agreement, image agreement, familiarity, and visual complexity. Journal of Experimental Psychology: Human Learning and Memory, 6(2), 174-215.

Spataro, P., Mulligan, N. W., \& Rossi-Arnaud, C. (2013). Divided attention can enhance memory encoding: The attentional boost effect in implicit memory. Journal of Experimental Psychology: Learning, Memory, and Cognition, 39(4), 1223-1231.

Ste-Marie, D. M., \& Jacoby, L. L. (1993). Spontaneous versus directed recognition: The relativity of automaticity. Journal of Experimental Psychology: Learning, Memory, and Cognition, 19(4), 777-788.

Troyer, A. K., \& Craik, F. I. (2000). The effect of divided attention on memory for items and their context. Canadian Journal of Experimental Psychology, 54(3), 161-171.

Tulving, E., \& Schacter, D. L. (1990). Priming and human memory systems. Science, 247(4940), 301-306.

Weeks, J. C., Biss, R. K., Murphy, K. J., \& Hasher, L. (2016). Face-name learning in older adults: A benefit of hyper-binding. Psychonomic Bulletin and Review, 1-7.

Weeks, J. C., \& Hasher, L. (2014). The disruptive - and beneficial - effects of distraction on older adults' cognitive performance. Frontiers in Psychology, 5, 133. 